\title{
Digital Microfluidics for Reconfigurable Antennas
}

\author{
Yasin Damgaci $^{1}$, Engin Cagatay ${ }^{2,3}$, Necmi Biyikli $^{2,3}$, Bedri Artug Cetiner $^{1}$ \\ ${ }^{1}$ Department of Electrical and Computer Engineering, Utah State University, Logan UT 84322-4120 U.S.A. \\ ${ }^{2}$ UNAM - National Nanotechnology Research Center, Bilkent University, Bilkent, Ankara 06800 TURKEY \\ ${ }^{3}$ Institute of Materials Science and Nanotechnology, Bilkent University, Bilkent, Ankara 06800 TURKEY
}

\begin{abstract}
Usage of fluids (microfluidic or otherwise) in antennas provides a conceptually easy reconfiguration mechanism in the aspect of physical alteration. However, a requirement of pumps, valves, etc. for liquid transportation makes the antenna implementations rather impractical for the real-life scenarios. This work reports on the theoretical calculations and experiments conducted to evaluate the electrowetting on dielectric (EWOD) driven digital microfluidics as a reconfiguration mechanism for antennas and $\mathrm{RF}$ circuits.
\end{abstract}

Index Terms- microfluidic, reconfigurable antenna, liquid metal, electrowetting.

\section{INTRODUCTION}

There is a high demand for antennas that can dynamically change their radiation properties with the rapid growth of wireless communication devices. Reconfigurable antennas are capable of changing the operating frequency, bandwidth, radiation pattern or polarization by different mechanisms integrated within the antenna aperture. These mechanisms can be divided into three groups: (i) usage of electronic components such as; radio frequency (RF)-switches or varactors; (ii) utilization of tunable materials and/or substrates, and (iii) direct mechanical/structural changes in antenna itself.

Fluidic systems have also been proposed for antenna reconfiguration which can be categorized as a subset for the mechanical/structural changes group. The use of fluids (microfluidic or otherwise) in antennas provides a conceptually easy reconfiguration mechanism. However, these types of antennas need external physical force (pumping, strain etc.) for dynamic reconfiguration.

Here, we propose electrowetting on dielectric (EWOD) driven digital microfluidics (DMF) as an alternative reconfiguration mechanism. DMF is different from continuous-flow microfluidics as it allows for precise control over individual droplets with no need for complex networks of connections, micro valves, or pumps.

\section{METHODS}

\section{A. Selecting a Liquid}

There are some new design requirements for digitalmicrofluidic antennas, which consist of combining the world of electromagnetics and digital microfluidics. Since EWOD deals with micro or nano-liter liquids, it is commonly used for designing droplet switches in the RF/microwave frequencies. With a single droplet, these components could be designed as an alternative for micro-electromechanical (MEMS) switches. For the antenna case, other metrics should be considered for successful reconfiguration performance like tuning speed, reliability, and bias circuit complexity. Depending on antenna design frequency, the amount of liquid introduces new challenges for the EWOD operation. Thus, the use of extensive volume of liquids should be avoided which requires an intensive use of electrodes and biasing lines. Choosing liquid is especially critical in the sense of electrowetting mechanism and electromagnetic compatibility. Although, liquid metals and liquid dielectrics have been both demonstrated for antenna applications, their dynamic manipulation with electrowetting plays a critical role. Most of the current EWOD applications are focused on the dielectric liquid manipulation, which might not be suitable for high frequency applications, due to high dielectric loses. Nanoparticle mixes are used for antenna designs, but their longterm reliability under electrostatic actuation is not explored fully. Electrolyte solutions could be considered as conductive, but their conductivities are too low for efficient radiation. Liquid metals are the naturally preferred option since most of the antenna types require metallic parts for radiation. Only a few metals are available in liquid form at room temperature. Mercury is mostly used in electrowetting because of it is availability even though it has a toxic nature. Non-toxic room temperature liquid metal alloys have been available, but thin oxide layer formation with air exposure needs to be controlled carefully for successful electrowetting actuation.

\section{B. Actuation Characterization of Liquid Metal Droplets}

Three different metallic liquids have been used throughout the experiments: Mercury $(\mathrm{Hg})$, Eutectic Gallium Indium (EGaIn) and Gallium Indium Tin alloy (Galinstan).

Mercury is a low-viscosity, Newtonian fluid (like water or oil), and does not oxidize readily (i.e., does not form a skin) under ambient conditions. Mercury brings advantages as a result of its inherent properties. For example, due to high surface tension $(435 \mathrm{mN} / \mathrm{m})$ mercury droplets act like a solid ball. Moreover, because of its low adhesion to metallic and dielectric surfaces, no residue is found when mercury is moved from one location to another, making it an ideal liquid metal. However, due to its toxicity, the use of mercury has been limited stemming from concerns about environmental hazards and health issues.

Eutectic Gallium-Indium (EGaIn) is an electrically conductive fluid metal alloy, consisting of 75 wt.\% Ga and 25 wt.\% In. Galinstan, which is also liquid at room temperature with melting point at $-19 \mathrm{C}$, is an eutectic alloy of $68.5 \% \mathrm{Ga}$, $21.5 \% \mathrm{In}$, and $10 \% \mathrm{Sn}$ by weight.

Severely fast oxidation of Galinstan and EGaIn in air poses a great difficulty in handling them, which becomes especially significant in microscale because of the large area-to-volume 
ratio, so that droplets might form a distinctively nonspherical shape, even at only $0.2 \%$ oxygen in a nitrogen-filled glove box. Therefore, although Galinstan is usually assumed to wet almost all surfaces, it is the oxidation of Galinstan that produces the appearance of wetting.

The first generation of test devices consisted of thermal evaporated 10/100 nm-thick $\mathrm{Cr} / \mathrm{Au}$ electrodes and $300 \mathrm{~nm}$ thick PECVD-grown $\mathrm{SiO}_{2}$ dielectric layer (Figure 1). Fabrication was performed on 4" fused silica or quartz wafers. The sole purpose of these devices with interdigitated fingers was to prove that the liquid metal droplets could be actuated and moved. A successful droplet actuation experiment with $>$ $80 \mathrm{~V}$ applied voltage was achieved for mercury and, actuation characteristics were analyzed with various device architectures. Experiments using Galinstan and EGaIn liquid droplets revealed that they form a skin of gallium oxide after even a brief exposure to air. Contact angles of Galinstan and EGaIn were measured to be 110 and 134, respectively. However, these values were not reliable and estimated contact angles depend on the shape of the droplets, since they had nonspherical shapes. Depending on these results mercury was used in several reconfigurable antenna designs

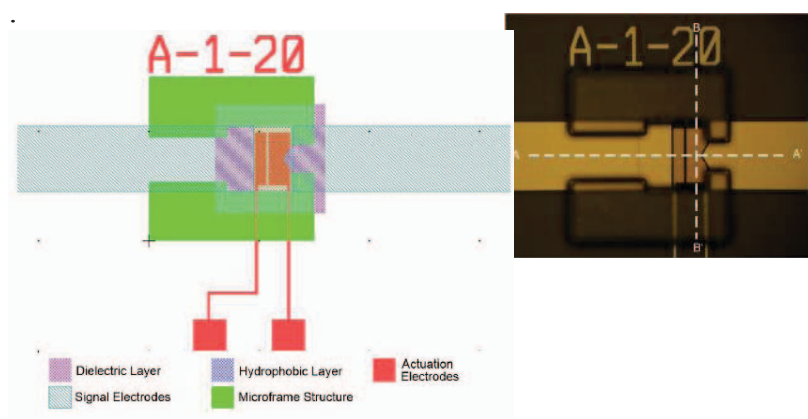

Fig. 1. Actuation test device for $\mathrm{Hg}$ droplet using EWOD.

\section{RECONFIGURABLE ANTENNA APPLICATIONS}

\section{A. Frequency Reconfigurable Slot Antenna}

The antenna presented here is a coplanar waveguide (CPW) fed annular slot antenna which is resonant at the frequency whose guided wavelength in the slot approximately corresponds to the mean circumference of the annular slot. (Fig. 2) shows the 3-D schematic of the MRA with an inset showing the magnified view of the integrated microfluidic chip. The basic antenna structure is a CPW fed annular slot built on a quartz substrate with permittivity $\varepsilon_{r}=3.9$, losstangent $\tan \delta=0.0002$, and height $=1 \mathrm{~mm}$.

The dimensions of the antenna and CPW of $50 \Omega$ characteristic impedances along with the transition regions have been determined for the targeted X-band operation (center resonance $\sim 12 \mathrm{GHz}$ ). The digital microfluidic chip, which consists of SU-8 based a cylindrical micro-chamber, micro-frames (4 columns) and EWOD platform with a mercury droplet placed in it, is located at the right side of the MRA center. In order to precisely control the dispensing, positioning and the spreading of the mercury droplet by EWOD, it is confined within the micro-frame structure, which also serves to avoid undesired gravitational effects. Moreover, a circular chamber design was used to house silicone oil droplet, which encapsulates the mercury droplet. This silicone oil serves for reducing actuation voltage for spreading the mercury droplet, reduces the uncertainty due to contact-angle hysteresis, and also provides the initial structure for ultimate packaging. Ultimately, the volume of the mercury droplet has been so determined $(1 \mu \mathrm{L})$ that not only the RF capacitive loading required for the desired frequency tuning range $(\sim 2$ $\mathrm{GHz}$ ) but also a simple EWOD platform can be obtained. Accordingly, the associated dimensions corresponding to these design features have been determined by full-wave EM analyses and are given in the caption of (Fig. 3).

As shown in (Fig.3), the RF ground and signal planes comprise the EWOD electrode. The dispensed mercury droplet sits on the Parylene dielectric layer, which is deposited on top of the high resistive TaN layer. This resistive layer is used to increase the isolation between the antennas RF signal and ground planes. It is important to note that the capacitive coupling occurs due to the mutual coupling between the

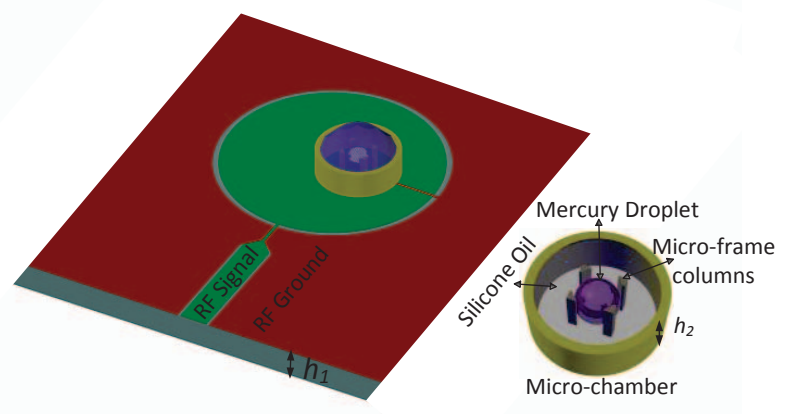

Fig. 2. 3-D schematic of the MRA integrated with a digital microfluidic chip with a magnified view of the chip.

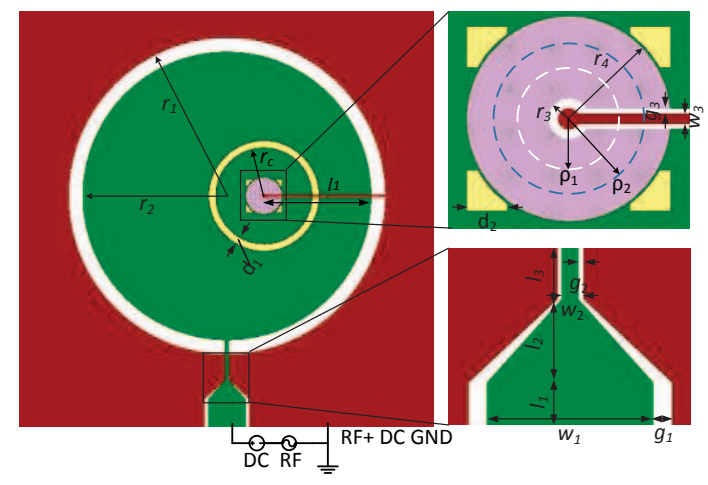

Fig. 3. Top view of the MRA with magnified views of the CPW feed and EWOD platform. $\mathrm{r} 1=4.1, \mathrm{r} 2=3.9, \mathrm{rC}=1.3, \mathrm{r} 3=0.1, \mathrm{r} 4=0.5$, w1 $=1$, w2 $=0.1$, $\mathrm{w} 3=0.05, \mathrm{~g} 1=0.115, \mathrm{~g} 2=0.0115, \mathrm{~g} 3=0.025,11=4.5,12=0.45,13=0.7$,

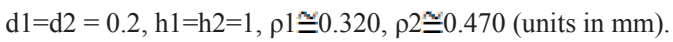

mercury droplet and RF signal plane. The antenna was fabricated on a 4-inch Quartz wafer $\left(\varepsilon_{r}=3.9\right)$ with a thickness of $1 \mathrm{~mm}$. Total antenna size is $20 \mathrm{~mm}$ x $20 \mathrm{~mm}$. $200 \mathrm{~nm}$ Tantalum Nitride (TaN) with a sheet resistivity $700 \mathrm{ohm} / \mathrm{sq}$ 
was deposited on the substrate. Deposition was made by reactive sputtering and patterned lithographically using a reactive ion etching. Antenna metal was formed by lift-off of a $20 \mathrm{~nm}$ Chromium (Cr), $1000 \mathrm{~nm}$ Copper $(\mathrm{Cu}), 20 \mathrm{~nm} \mathrm{Cr}$ and $30 \mathrm{~nm}$ Nickel (Ni) metals. To this end, a thin layer of nickel (Ni) must be deposited at to avoid mercury reaction with other metals. On top of the multilayer metal, a $1 \mu \mathrm{m}$ thick dielectric layer of Parylene $\mathrm{C}$ was deposited at room temperature by vapor deposition, which was then patterned by reactive-ionetch (RIE) using Oxygen $\left(\mathrm{O}_{2}\right)$ plasma. The height of SU-8 microframes $\quad(\sim 1 \mathrm{~mm})$ was determined by the typical diameter $(\sim 1 \mathrm{~mm})$ of the liquid metal droplets. To obtain such an ultra-thick microframe structures with a single spin, SU-8 2100 was deposited with a different recipe than the data provided by manufacturer. Low spin coating speed and soft baked with a high temperature was used. To increase the adhesion, OmniCoat was used. The temperatures for the softbaking process and post-exposure baking are $120{ }^{\circ} \mathrm{C}$ and 55 ${ }^{\circ} \mathrm{C}$, respectively which prevents the structures from cracking and distorting during developing and hard-baking processes. Temperature was always ramped up or down with a rate of 1 ${ }^{\circ} \mathrm{C} / \mathrm{min}$ from $50{ }^{\circ} \mathrm{C}$. Step exposure with each step consisting of a $60 \mathrm{~s}$ exposure and a $30 \mathrm{~s}$ delay was used After 4 hours of post-exposure bake, the features were developed with slow agitation. Finally, a $\sim 1 \mathrm{~mm}$-diameter mercury droplet was placed in each micro frame manually. In dispensing the droplets, Gilson Microman ${ }^{\circledR}$ micropipette was used.

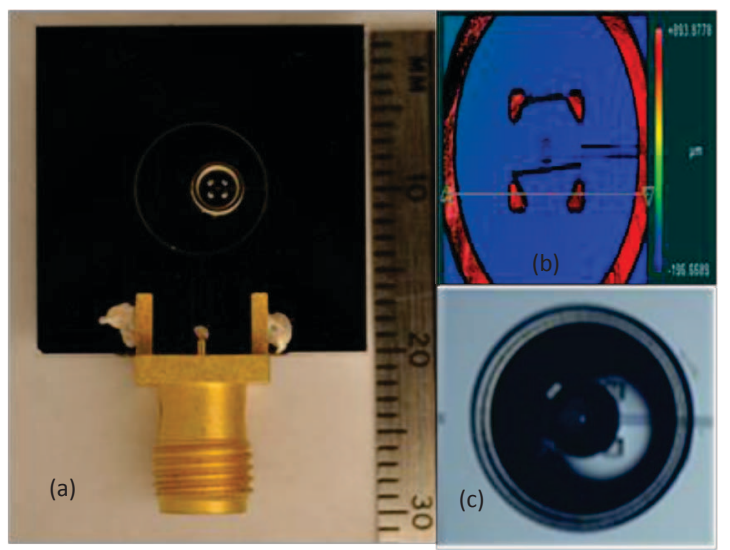

Fig. 4. The photographs of (a) the top view of the fabricated MRA (b) optical profilometer surface map of microfluidic chip with SU8 thickness $1090 \mathrm{um}$ and (c) mercury + silicone oil filled microfluidic chip

The frequency response of the fabricated antenna prototype has been measured and the results were compared with those of the simulated ones. To perform the RF measurements, a $3.5 \mathrm{~mm}$ of the CPW feed line by using a conductive epoxy, as shown in (Fig. 4). The prototype was then connected to a fully-calibrated single port of a vector network analyzer (VNA). To spread the mercury droplet by EWOD, a DC actuation voltage was supplied by a separate voltage source, which was superimposed with the RF source signal provided by the VNA (see Fig. 3). The total signal was applied to the CPW feed line via bias tees, which are used to prevent high
DC voltages from going to the VNA. The results for antenna reflection coefficients are shown in (Fig. 5), where the tuning in frequency in response to the spread of the mercury droplet is clearly observed. When the mercury droplet is in its unactuated state, the center frequency of operation is at around $13 \mathrm{GHz}$ as confirmed by both from measurements and simulations as shown in (Fig. 5). obtained by full-wave EM simulations for both modes of operations. The integrity of the radiation properties (gain values and shape of the patterns) is maintained over the reconfigurable frequency band $(\sim 11-13$ $\mathrm{GHz})$.
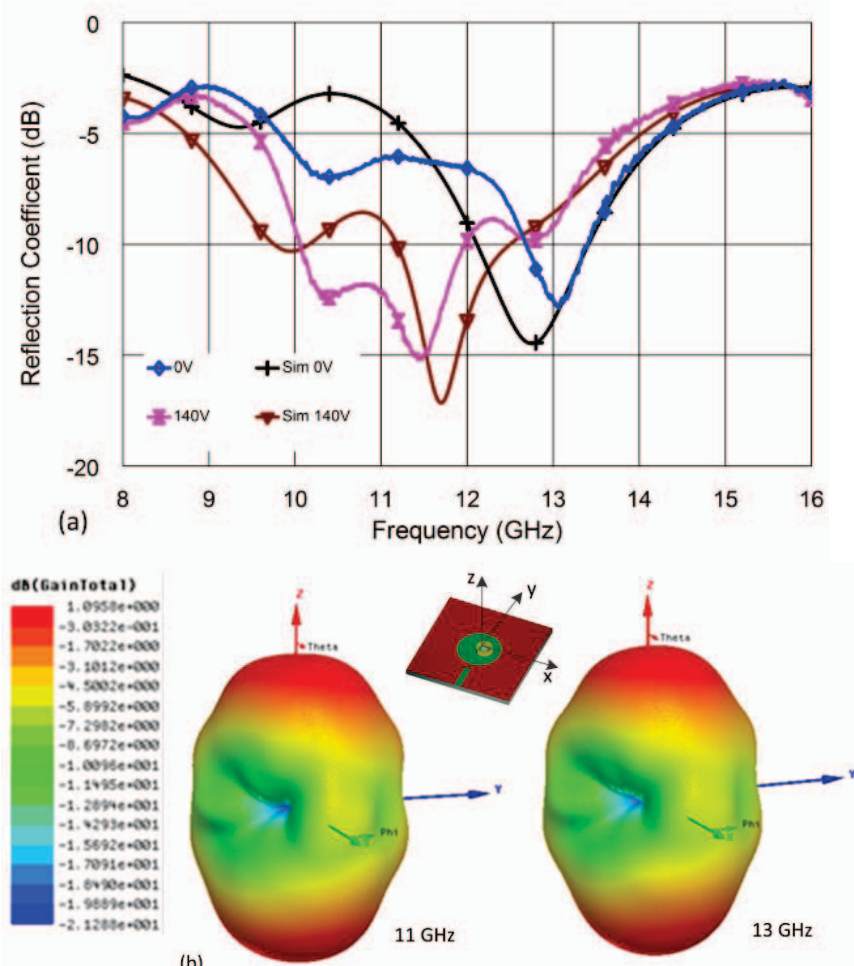

Fig. 5. Measured and simulated reflection coefficients and (b) simulated 3-D radiation patterns (realized total gain) corresponding to $f=11 \mathrm{GHz}$ (ON state) and $f=13 \mathrm{GHz}$ (OFF state) modes of operations

\section{B. Frequency Reconfigurable Zeroth-Order Resonator Antenna}

This approach is based on the unique metamaterial transmission line structures, which are leading to antenna miniaturization. Coplanar waveguide $(\mathrm{CPW})$ metamaterial transmission lines (TLs) were designed and used as digital microuflidic platforms, where tunable resonance frequency was demonstrated for radio frequency $(\mathrm{RF}) /$ microwave applications. The maximum tuning range achieved was around $400 \mathrm{MHz}$ (from $3.51 \mathrm{GHz}$ to $3.92 \mathrm{GHz}$ ) with a DC actuation voltage of $110 \mathrm{~V}$.

A Capacitor loaded CPW TL was used as an EWOD stage by simply adding a dielectric layer on the top of the TL metal layer. Fig. 6 shows the unit cell geometry along with the dimensions of critical design parameters. This structure has an 
interdigitated finger like pattern which creates the left-handed capacitance, $C_{L}$ and also serves as the actuation electrodes for EWOD. The interdigitated section is covered by a Parylene/Teflon dielectric layer, on top of which a liquid metal (mercury) droplet is placed. Fig. 6 depicts the cross section (A-A') view of the structure and also shows the EWOD phenomenon taking place. By using the EWOD phenomenon, this droplet is spread by DC actuation voltage applied between input and output ports of the structure as shown in Fig. 6. This movement of the liquid metal droplet increases its base contact area, which in turn changes (increases) the effective left-handed capacitance, $C_{L}$. Thus the resonant frequency is changed (decreased) resulting in the desired tuning.

Fig. 7 is used to describe the microfabrication processes, where 4-inch Quartz wafer $\left(\varepsilon_{r}=3.9\right)$ with a thickness of 0.525 $\mathrm{mm}$ is used as a substrate material. For the metal layers of the CPW TL, a $20 \mathrm{~nm}$ Chromium (Cr), $200 \mathrm{~nm}$ Copper $(\mathrm{Cu})$, $20 \mathrm{~nm} \mathrm{Cr}$ and $30 \mathrm{~nm}$ Nickel (Ni) metals were electron-beam (e-beam) evaporated successively and patterned by lift-off technique. This multilayer metal also serves for the EWOD actuation electrodes. On top of the multilayer metal, a $1 \mu \mathrm{m}$ thick dielectric layer of Parylene $\mathrm{C}$ was deposited at room temperature by vapor deposition, which was then patterned by reactive-ion-etch (RIE) using Oxygen $\left(\mathrm{O}_{2}\right)$ plasma. In addition

to Parylene $\mathrm{C}$, another dielectric layer of hydrophobic Teflon AF with a thickness of $\sim 80 \mathrm{~nm}$ was spun coated to minimize contact angle hysteresis improving the robust repeatable operation. After coating, Teflon AF layer was baked at $200{ }^{\circ} \mathrm{C}$ for 2 hours. Finally, this hydrophobic Teflon AF film was patterned by $\mathrm{O}_{2}$ plasma to define an opening of a circular-shape at the center, which was, subsequently, filled by an additional $20 \mathrm{~nm} / 30 \mathrm{~nm} \mathrm{Cr} / \mathrm{Au}$ layer deposited by Ebeam evaporation and patterned by lift-off. This circular pattern with a $100 \mu \mathrm{m}$ diameter at the center acts as an anchor providing a mechanical stability for the manually dispensed mercury droplet of $1 \mathrm{~mm}$ diameter. In dispensing the droplets Gilson Microman ${ }^{\circledR}$ micropipette was used.

The photographs of a fabricated device are shown in Fig. 8. The scattering parameter measurements of the fabricated CPW TLs have been performed by using Agilent 8722ES network analyzer with a 2-port Short Open Load Transmission (SOLT) calibration in the $50 \mathrm{MHz}-12 \mathrm{GHz}$ frequency band. DC actuation voltage was supplied by a separate voltage source, which was applied to the terminals of $\mathrm{RF}$ input and output ports via bias tees as shown in Fig. 2. In this measurement set- up, the DC voltage was superimposed with the RF source signal provided by the network analyzer, where the bias tees were used to prevent high DC voltages going into network analyzer. In order to observe the reversible operation of the fabricated devices, the measurements started by applying the DC voltages in increments of $10 \mathrm{Vs}(10 \mathrm{~V}, 20$ $\mathrm{V}, 30 \mathrm{~V} \ldots$ and $110 \mathrm{~V})$, where the DC source was turned off at each step. We clearly observed that the spread mercury droplet returned back to its original non-spread stage every time the source was turned off. Fig. 9 shows the zeroth-order resonance frequencies for different actuation voltages $(0,60$,
90, 110V). As seen from Fig.5, the maximum tuning range in excess of $400 \mathrm{MHz},(3.92$ to $3.51 \mathrm{GHz})$ is obtained.

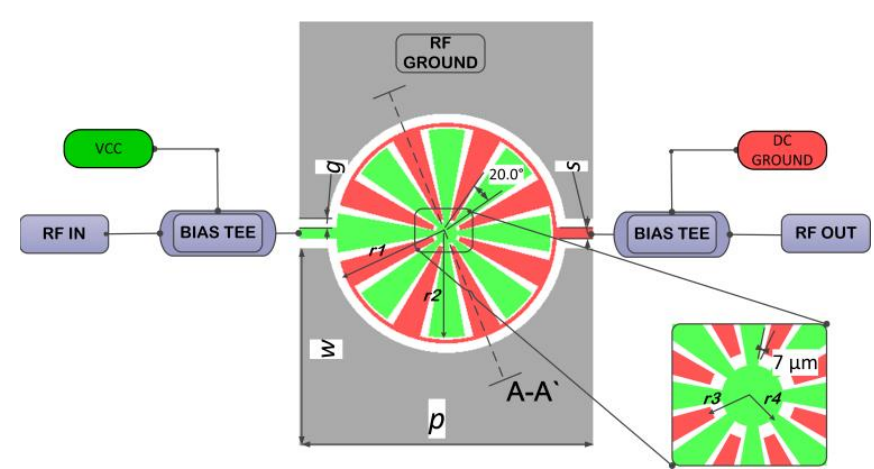

Fig. 6. The layout and test schematic of the tunable CPW metamaterialinspired TL. $p=2, w=1.395, s=g=0.07, r 1=0.75, r 2=0.7, r 3=0.1, r 4=0.05$ (in $\mathrm{mm}$ )

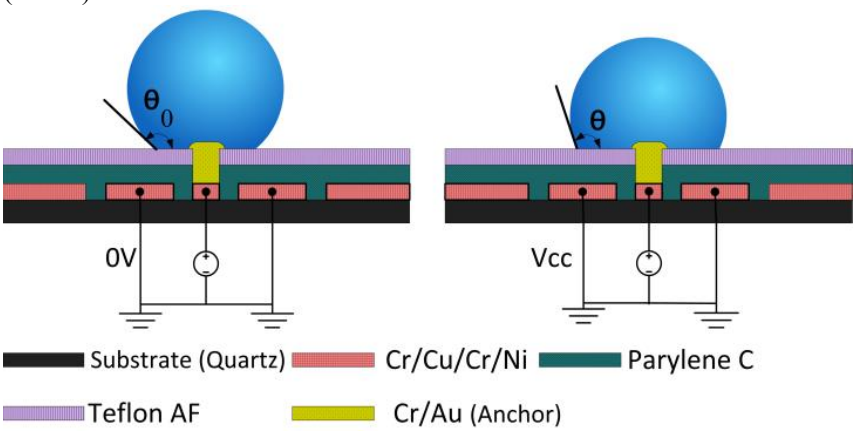

Fig. 7. Cross section (A-A') view of the structure and EWOD principle.
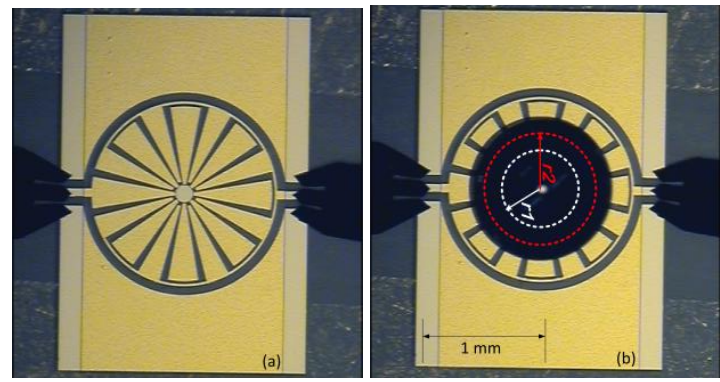

Fig. 8. The photographs of a fabricated device (a) before and (b) after mercury droplet dispensing. The boundaries of the base contact areas are illustrated with white and red dash lines before and after EWOD spreading, respectively $\left(r_{2}-r_{l} \sim 150 \mu \mathrm{m}\right)$.

The $\mathrm{n}=0$ resonance mode supported by a capacitor loaded CPW TL resonator is used to realize infinite wavelength resonant antennas with monopolar radiation patterns with the concept introduced last section. The infnite wavelength resonant antenna can be made arbitrarily small and single droplet could be used for reconfiguration. In the case of shortcircuit boundary conditions, the resonant frequency is determined by the series resonance of the unit cell. More unit cells can also be used to enhance the directivity of the proposed antenna. Capacitor-loaded CPW is short-circuited through a short section of CPW line of relatively higher characteristic impedance. This CPW section is needed to 
compensate for the degradation of input matching condition at the series resonance when fewer unit cells are used.

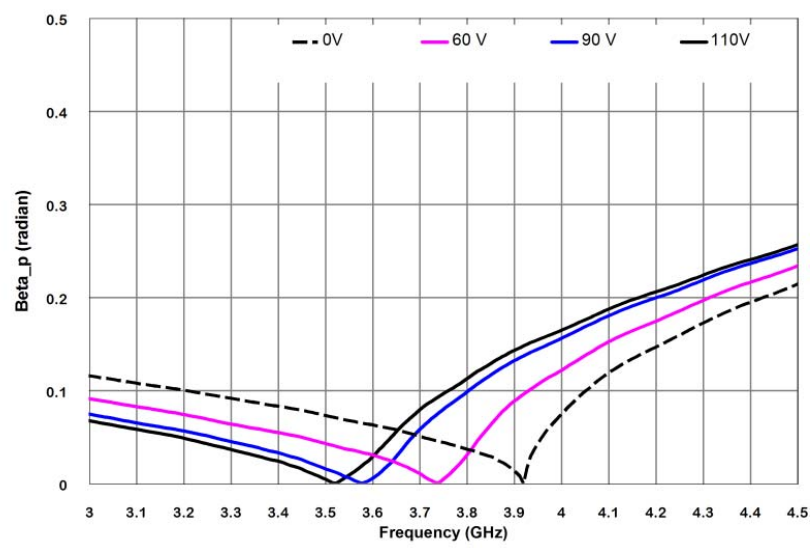

Fig. 9. Calculated propagation constant of the unit cell structure
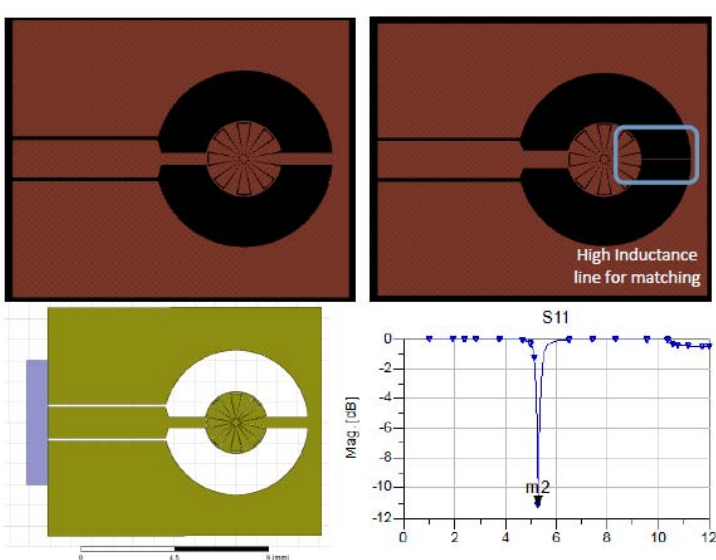

Fig. 10. ADS and HFSS models of the proposed antenna and reflection coefficient after matching.

\section{CONCLUSIONS}

Reconfigurable antennas consist of two main building blocks: radiation elements and reconfiguration mechanisms. In this work, development of new reconfiguration technology is addressed. Electrowetting on dielectric (EWOD) based digital microfluidics has been demonstrated as a novel reconfiguration technique for antennas. Theoretical and experimental proof is used to show that this technique allows iscrete volumes of liquid to be precisely controlled as independent units within microstructures which can be integrated with antennas.

\section{ACKNOWLEDGMENT}

This work was supported in part by the National Institute of Justice, Office of Justice Programs, US Department of Justice, under Award \# 2009-SQ-B9-K005, by National Science Foundation under Award \#1040435, by Utah Governor's office Center of Excellence, and by TUBITAK BIDEB. UNAM - National Nanotechnology Research Center is supported by the Turkish Ministry of Development. N.B. acknowledges Marie Curie International Reintegration Grant (NEMSmart, PIRG-GA-2009-249196)

\section{REFERENCES}

[1] Yasin Damgaci and Bedri A. Cetiner, "A frequency reconfigurable antenna based on digital microfluidics," Lab Chip, 2013,13, 2883-2887

[2] Damgaci, Y.; Cetiner, B.A., "Tunable Zeroth-Order Resonance in Metamaterial-Inspired Transmission Lines Based on Electrowetting on Dielectric," Microwave and Wireless Components Letters, IEEE, vol.23, no.8, pp.421,423, Aug. 2013

[3] Engin Cagatay, Mehmet Alican Noyan, Yasin Damgaci, Bedri A. Cetiner, and Necmi Biyikli, "Fabrication and characterization of liquid metal-based micro-electromechanical DC- contact switch for RF applications", Microtech Conference \& Expo 2012, Santa Clara, CA

[4] E. Cagatay, Y. Damgaci, B. A. Cetiner, and N. Biyikli, "Actuation of Liquid Metal Droplets Using Electrowetting-on-Dielectric (EWOD) for Reconfigurable Antenna Applications", MRS Fall Meeting, Boston, MA, (2011).

[5] S. Cheng, A. Rydberg, K. Hjort, and Z. Wu, "Liquid metal stretchable unbalanced loop antenna," Applied Physics Letters, vol. 94, no. 14, p. 144103, 2009.

[6] J.-H. So, J. Thelen, A. Qusba, G. J. Hayes, G. Lazzi, and M. D. Dickey, "Reversibly deformable and mechanically tunable uidic antennas," Advanced Functional Materials, vol. 19, no. 22, 2009.

[7] P. Sen and C.-J. Kim, "A fast liquid-metal droplet microswitch using EWOD-driven contact-line sliding," J. Microelectromech. Syst., vol. 18 no. 1, pp. 174-185, Feb. 2009.

[8] Z. L. Wan, H. J. Zeng, and A. D. Feinerman, "Area-tunable micromirror based on electrowetting actuation of liquid-metal droplets," Appl. Phys. Lett.,vol. 89, no. 20, p. 201 107, Nov. 2006. 